\title{
Shiga toxin induces lipid compression: a mechanism for generating
} membrane curvature

\author{
Erik B. Watkins ${ }^{1^{*}}$, Jaroslaw Majewski ${ }^{2,3,4}$, Eva Y. Chi ${ }^{4}$, Haifei Gao ${ }^{5}$, Jean-Claude Florent ${ }^{5}$, Ludger Johannes ${ }^{5^{*}}$ \\ ${ }^{1}$ MPA-11: Materials Synthesis and Integrated Devices, Los Alamos National Laboratory, Los Alamos, New Mexico 87545, USA. \\ ${ }^{2}$ Theoretical Biology and Biophysics, Los Alamos National Laboratory, Los Alamos, New Mexico 87545, USA. \\ ${ }^{3}$ Division of Molecular and Cellular Biosciences, National Science Foundation, Alexandria, Virginia, USA. \\ ${ }^{4}$ Department of Chemical and Biological Engineering and the Center for Biomedical Engineering, University of New Mexico, Albuquerque, \\ New Mexico 87131 , USA. \\ ${ }^{5}$ Institut Curie, PSL Research University, Cellular and Chemical Biology unit, U1143 INSERM, UMR3666 CNRS, 26 rue d'Ulm, 75248 \\ Paris Cedex 05, France.
}

KEYWORDS: Biomembrane, Shiga toxin, membrane curvature, endocytosis.

\section{SUPPORTING INFORMATION:}

\author{
$\underline{\text { Introductory comments }}$
}

While it has been shown that binding of $\mathrm{STxB}$ to $\mathrm{Gb} 3$ receptors triggers membrane mechanical events that lead to the biogenesis of tubular endocytic pits, many of the mechanistic aspects on how this is achieved still need to be clarified. ${ }^{1}$ One factor contributing to the membrane reorganization mechanism may rely on the clustering of toxin molecules on the membrane surface, resulting in nanoscale regions with high bound protein concentrations. It was recently shown that increasing the distance separating Gb3's carbohydrate from its ceramide by polyethylene glycol (PEG) linkers led to a loss of STxB clustering and to an inhibition of its endocytic uptake into cells. ${ }^{2}$

We report here the effect of these linkers on the structural reorganization of membrane lipids induced by STxB binding, and discuss how this relates to the mechanism for the initiation of tubular endocytic pit formation. Using $x$-ray reflectivity (XR) and grazing-incidence $x$-ray diffraction (GIXD), we investigated how STxB binding to Gb3 in model lipid membranes alters the lipid order, and how flexible PEG linkers ( $n=1,3$, and 7 monomer lengths) between the Gb3 carbohydrate and ceramide moieties modulate these effects. Monolayers were composed of DPPE and Gb3 and maintained at a surface pressure of $30 \mathrm{mN} / \mathrm{m}$ and $23^{\circ} \mathrm{C}$. To approximate the membrane composition and local environment of bound toxin clusters, these studies were performed at high Gb3 receptor concentrations (20\%) and high coverage of bound $\mathrm{STxB}$, as one would expect to find at sites of endocytic pit construction. Molecular dynamics simulations indeed indicated that a high Gb3 concentration of up to $30 \mathrm{~mol} \%$ is found in the membrane region beneath bound $\mathrm{STxB} .^{3,4}$

$\underline{\mathrm{XR}}$ measurements and electron density distributions before and after STxB binding

$\mathrm{X}$-ray reflectivity (XR) from the air-water interface was used to obtain high resolution electron density profiles of the lipid monolayer and monolayer/protein systems studied. Oscillations in the XR signal originate from interference between $\mathrm{x}$-rays reflected from layers within the system with different electron densities (Figure S-1). In a simplified approximation, the frequency of oscillations corresponds to a length scale, $d$, in the system where $d=2 \pi / \Delta q_{z}$ where $\Delta q_{z}$ is the $q$ spacing between minima or maxima of the oscillation. More detailed structural information can be extracted from the XR measurements by modelling the data using the Parratt or Abeles formalism.,

Electron density distributions, obtained from fitting the XR data, corresponding to DPPE:Gb3 ${ }_{n}$ monolayers for the full range of PEG linker lengths studied is presented in Figure S-2. For the DPPE monolayer, the center of the head groups corresponds to the maximum electron density at $\mathrm{z}=0$. The shoulder to the left of this maximum corresponds to the hydrocarbon tails, and the distribution plateaus to the right at the electron density of water. Compared to pure DPPE, the 80:20 DPPE:Gb30 monolayer exhibits electron density extension into the water corresponding to the Gb30 carbohydrate. For higher $n$, the electron density corresponding to the carbohydrate extends further from the membrane plane with an extension up to 25 $\AA$ into the water for $n=7$. 


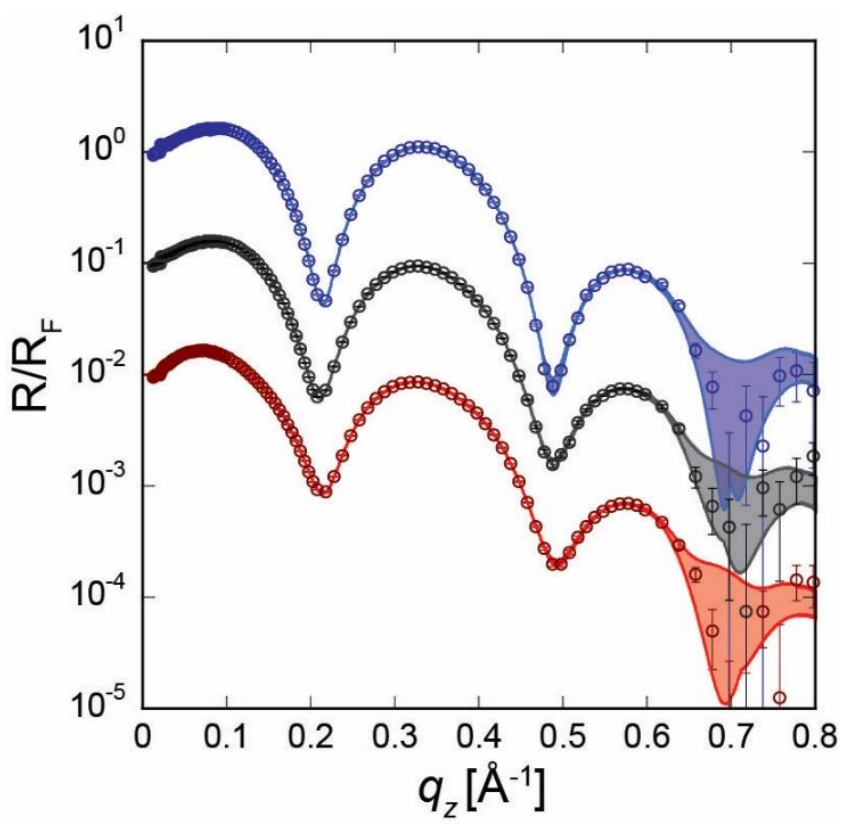

Figure S-1. Representative XR data (symbols with error bars denoting standard deviation) and the corresponding fits (shaded regions) for pure DPPE (blue), 80:20 mol\% DPPE:Gb3 ${ }_{0}$ before (gray), and after binding of $\mathrm{STxB}$ (red) monolayers at $30 \mathrm{mN} / \mathrm{m}$ at $20^{\circ} \mathrm{C}$. Data is offset

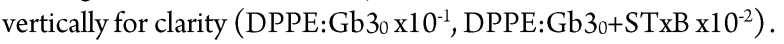

Some further structural variations between the monolayer structures containing different $\mathrm{Gb} 3$ receptors can be observed in both the head group and hydrocarbon tail regions. The $\mathrm{Gb} 3_{n}$ monolayers exhibits a lower head group maximum than DPPE, presumably due to the lower electron density of the Gb3 polar head group. There also appears to be a slightly reduced electron density of the hydrocarbon tails for DPPE:Gb3 ${ }_{n}$ compared to DPPE. This may be explained by reduction of the electron density caused by disordering of regions of the tail packing by the unsaturation in the Gb3 tail. In addition to model independent fitting, we analyzed the data using a box model approach to test these hypotheses. This approach allowed us to compare the following models to describe the monolayer structure: (1) tail and head densities of DPPE and DPPE:Gb3 monolayers were constrained to be the same; (2) tail densities of DPPE and DPPE:Gb3 monolayers were constrained to be the same but head densities were allowed to vary; (3) head densities of DPPE and DPPE:Gb3 monolayers were constrained to be the same but tail densities were allowed to vary; (4) neither tail nor head densities were constrained to be the same. Using these four models, we simultaneously co-refined the fits to pure DPPE and the four DPPE:Gb3 monolayers. As expected, the lowest $\chi^{2}$ value of 19.5 was obtained for model (4) which had the largest number of free parameters. In contrast, model (1), with the least amount of free parameters, had the highest $\chi^{2}$ with a value of 24.0. A $\chi^{2}$ value of 20.5 was obtained for model (2), and a $\chi^{2}$ value of 23.5 was obtained for model (3). These results show that variation in tail density between pure DPPE and DPPE:Gb3 monolayers does not significantly impact the goodnessof-fit while variation in the head density does. This suggests that, in comparison to the variation in head group electron density, the relatively small variation in tail density obtained in the model independent fitting approach presented in Figure S-2A is not statistically significant. On the other hand, the reduced electron density of the head group region in DPPE:Gb3 monolayers compared to pure DPPE monolayers is significant and can be attributed to the lower electron density of the Gb3 polar head group.
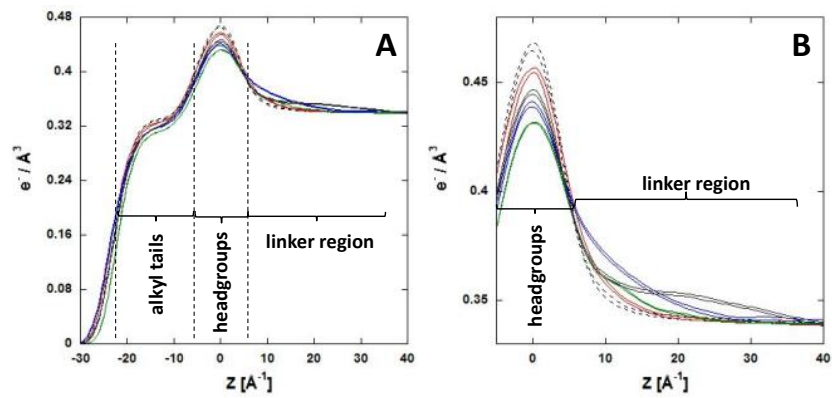

Figure S-2. (A) Electron density distributions obtained from XR analysis of pure DPPE and 80:20 mol\% DPPE:Gb3 ${ }_{n}$ monolayers at $30 \mathrm{mN} / \mathrm{m}$ at $20^{\circ} \mathrm{C}$. The electron densities are represented as "ribbons" (regions between the same color lines). Black dashed lines: pure DPPE, red solid lines: $n=0$, green solid lines: $n=1$, purple solid lines: $n=3$, and gray solid lines: $n=7$. Maxima of electron densities, corresponding to the center of the head group region, were set to $z=0$. (B) Magnification of the electron densities in the head group and linker regions. For higher $n$, electron density contributions from the carbohydrate extend further from the membrane.
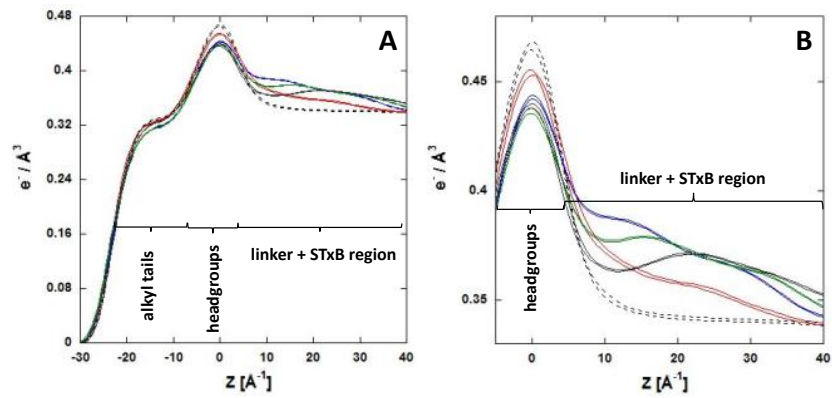

Figure S-3. (A) Electron density distributions obtained from XR analysis of pure DPPE and 80:20 mol\% DPPE:Gb3 $n$ monolayers with bound $\mathrm{STxB}$ at $30 \mathrm{mN} / \mathrm{m}$ at $20^{\circ} \mathrm{C}$. The electron densities are represented as "ribbons" (regions between the same color lines). Black dashed lines: pure DPPE, red solid lines: $n=0$, green solid lines: $n=1$, purple solid lines: $n=3$, and gray solid lines: $n=7$. Maxima of electron densities, corresponding to the center of the head group region, were set to $z=0$. (B) Magnification of the electron densities in the head group and linker regions.

After STxB binding, significant changes to the electron density profiles corresponding to the bound protein were observed (Figure S3 ). These profiles were used to extract the protein distributions presented in Figure 1c of the main text, and the average separation between the STxB proteins and the membrane plane which is dis- 
cussed in a following section. It was not possible to resolve the carbohydrate penetrating into the protein's receptor pockets independently from the $S T x B$ protein signal. From the XR measurement, we obtain a 1-D electron density profile normal to the monolayer. The region in this profile where the carbohydrate penetrates the STxB binding pocket is a superposition of the electron densities of the protein, carbohydrate, water that is between neighboring proteins, and water hydrating both carbohydrates and proteins. It is extremely difficult to deconvolute the individual contributions to the electron density in this region, especially for the case of the carbohydrate which is contributing much less electron density than the protein and water. Complicating this further is the fact that the orientation of the Gb3 carbohydrates changes after protein binding.

\section{GIXD measurements of lipid APM before and after STxB binding}

In all cases, the lipid diffraction resulted in a single GIXD Bragg peak corresponding to a superposition of $(1,0),(0,1)$, and $(-1,1)$ Bragg reflections and 2-D hexagonal packing. In the absence of $S T x B$, there were no significant differences in the GIXD Bragg peak positions, and therefore APM, for pure DPPE and the 80:20 DPPE:Gb3 ${ }_{n}$ monolayers (Table S-1). This indicates that neither the incorporation of 20 mol\% Gb3 nor the addition of flexible linkers between the Gb3 carbohydrates and ceramide backbone had any significant impact on nanoscale lipid tail order. However, after STxB binding the positions of the Bragg diffraction peaks showed significant changes as a function of linker length. For $n=0,1,3, \mathrm{STxB}$ binding resulted in peaks shifted to smaller $q_{x y}$ indicating that $\mathrm{STxB}$ induced APM condensation of the lipid tails. No significant change in the peak position was detected after $\mathrm{STxB}$ binding to monolayers containing $\mathrm{Gb}_{7}$, indicating that lipid APM was not altered in this condition. Furthermore, the absence of any significant peak broadening indicated that STxB binding impacted all lipids in the monolayer. This suggests that there is only one population of lipid order contributing to the diffraction and, since there is no contribution to the diffraction from non-condensed lipids, lipid APM condensation occurs even in areas of the monolayer that are not in the immediate vicinity of a bound protein.

Table S-1: Lipid monolayer GIXD packing parameters

\begin{tabular}{|c|c|c|c|}
\hline $\begin{array}{c}\text { Monolayer } \\
30 \mathrm{mN} / \mathrm{m} \\
23^{\circ} \mathrm{C}\end{array}$ & $\begin{array}{c}\text { Unit cell } \\
a \\
\pm 0.002 \AA\end{array}$ & $\begin{array}{c}\text { Area per } \\
\text { molecule } \\
\pm 0.05 \AA^{2}\end{array}$ & $\begin{array}{c}\text { Coherence Length } \\
L_{x y} \\
\pm 30 \AA\end{array}$ \\
\hline DPPE & 4.851 & 40.76 & 185 \\
\hline DPPE:Gb30 & 4.850 & 40.75 & 203 \\
+STxB & 4.842 & 40.61 & 197 \\
\hline DPPE:Gb3 $3_{1}$ & 4.852 & 40.77 & 258 \\
+STxB & 4.840 & 40.58 & 228 \\
\hline DPPE:Gb3 3 & 4.853 & 40.79 & 239 \\
+STxB & 4.833 & 40.46 & 258 \\
\hline DPPE:Gb3 37 & 4.851 & 40.76 & 170 \\
+STxB & 4.852 & 40.77 & 183 \\
\hline
\end{tabular}

$L_{x y}$ corresponds to the average lateral dimension of positional registry of lipid tails.
In general, $\mathrm{ST} \times \mathrm{B}$ binding decreased the lipid APM throughout the entire monolayer. To fully interpret the measured trends in APM condensation, the degree of linker flexibility was taken into account as described in the main text. Another contributing factor that may influence the magnitude of the lipid condensation effect is the quantity of bound protein, even though for all linker lengths there was sufficient binding to impact all the lipids in the monolayer. An increasing quantity of bound protein from $n=0$ to $n=3$ correlates with the increasing APM condensation for these linkers. However, binding to $n=7$ linkers also yielded high protein coverage but there was no corresponding APM condensation.

Table S-2: STxB protein GIXD packing parameters

\begin{tabular}{|c|c|c|c|c|}
\hline $\begin{array}{c}\text { Monolayer } \\
30 \mathrm{mN} / \mathrm{m} \\
23^{\circ} \mathrm{C}\end{array}$ & $\begin{array}{c}\text { Unit cell } \\
a \\
\pm 0.5 \AA\end{array}$ & $\begin{array}{c}\text { Area per } \\
\text { molecule } \\
\pm 90 \AA^{2}\end{array}$ & $\begin{array}{c}\text { Coherence } \\
\text { Length, } L_{x y} \\
\pm 150 \AA\end{array}$ & $\begin{array}{c}\text { Relative } \\
\text { intensity } \\
\pm 0.25\end{array}$ \\
\hline $\begin{array}{c}\text { DPPE:Gb30 } \\
+ \text { STxB }\end{array}$ & 62.5 & 3385 & 760 & 0.84 \\
\hline $\begin{array}{c}\text { DPPE:Gb3 } \\
\text { +STxB }\end{array}$ & 62.3 & 3361 & 644 & 1.00 \\
\hline $\begin{array}{c}\text { DPPE:Gb3 } 3 \\
+ \text { STxB }\end{array}$ & 63.3 & 3465 & 619 & 0.55 \\
\hline $\begin{array}{c}\text { DPPE:Gb3 } \\
+ \text { STxB }\end{array}$ & - & - & - & 0.00 \\
\hline
\end{tabular}

$L_{x y}$ corresponds to the average lateral dimension of protein clusters.

\section{Quantifying bound STxB APM, coverage, and clustering}

A qualitative examination of the data showed that $\mathrm{ST} \mathrm{xB}$ binding was efficient and resulted in high membrane coverage for all linker lengths (see Figure 1c in the main text). However, the protein distributions extracted by subtracting the electron density profiles before and after STxB binding do not account for the electron contributions of the water displaced by the protein volume, resulting in an under-estimation of the true electron density distribution of the protein. Nonetheless, qualitative comparisons between the distributions can be drawn. In this section, XR and GIXD results are combined to enable quantitative calculations of small variations in the fraction of monolayer with $\mathrm{STxB}$ bound to it. Additionally, this analysis provided the fraction of proteins clustered in well-ordered domains within the STxB layer. The results of these analyses are presented in Figure 2 of the main text.

The monolayer fraction with bound $\mathrm{STxB}$, or protein coverage, was determined by dividing the APM of $\mathrm{STxB}$ in ordered clusters obtained from GIXD by the average APM of STxB obtained by XR. To obtain the average APM from XR, the electron density distributions corresponding to bound $\mathrm{STxB}$ in the difference profiles (main text, Figure 1c) were integrated, yielding the average electron areal density $\left(\mathrm{e}^{-} / \AA^{2}\right)$. However, the difference distributions do not directly provide the electron density distribution of the $\mathrm{STxB}$ protein due to over subtraction of the quantity of water displaced by the protein and, to a much smaller degree, the PEG linkers. To account for this, the water volume displaced by a single $\mathrm{STxB}$ was approximated as $46.5 \mathrm{~nm}^{3}$ by summing the individual amino acid volumes in the protein's sequence. ${ }^{7}$ Since the water displaced by the PEG linkers was 
orders of magnitude smaller $\left(\sim 0.065 \mathrm{~nm}^{3}\right.$ per monomer $)$, this volume was not explicitly accounted for. The total number of STxB electrons $(20,530)$ was adjusted by the displaced water electrons $(15,500)$ to yield 5,030 electrons associated with each STxB protein in the difference profile. Average APMs of STxB were calculated by dividing the adjusted electron number by the electron areal density obtained from the difference profiles. Finally, dividing the APM obtained from GIXD $\left(34 \mathrm{~nm}^{2}\right)$ by the average STxB APM yielded average values of protein coverage as a function of linker length (see main text Figure 2, black circles). Errors on these values correspond to the range of values in the difference electron density distribution ribbons of the proteins.

While STxB binding was efficient for all PEG linker lengths, the combination of XR and GIXD enabled small but measurable differences in membrane coverage to be determined. $\mathrm{STxB}$ coverage ranged from approximately $25-50 \%$ of the monolayer surface with potential trends for increasing coverage between $n=0$ to $n=3$, followed by a decrease for $n=7$ linkers. While the change in coverage may reflect sample-to-sample variations, we speculate on some possible mechanisms for the observed differences. The increase in coverage for short linkers may be due to greater exposure and mobility of the $\mathrm{Gb} 3$ carbohydrate residues facilitating binding to the STxB receptor sites. ${ }^{8}$ The decrease for $n=7$ linkers may be due to more disordered in-plane packing of the $\mathrm{STxB}$ proteins inhibiting high coverage. In a previous study, fluorescence intensity measurements were used to quantify $S T x B$ binding to the same modified Gb3 receptors used here and indicated a subtle decrease in the quantity of bound protein with increasing PEG linker length. ${ }^{2}$ While it has not been demonstrated that the variation in protein binding were statistically significant in either study, the results are in apparent contradiction. This inconsistency, if statistically significant, may be attributed to a variety of factors including differences in the membrane compositions and the factor of 10 difference in protein concentrations used in the two studies.

A combination of XR and GIXD results was also used to estimate the fraction of ordered STxB clusters within the entire protein layer. For all the cases where diffraction from the protein was observed, a single peak indicated clustering of the protein in ordered domains with hexagonal packing of proteins and an area per molecule (APM) of approximately $34 \mathrm{~nm}^{2}$. Using the Scherrer equation, which relates the broadening of diffraction peaks to the size or the diffracting domain, the diameter of the protein clusters were estimated to be $60 \pm$ $15 \mathrm{~nm}$ which corresponds to roughly 50-150 STxB proteins. ${ }^{9}$ The structural parameters resulting from the analysis of the protein diffraction are shown in Table S-2. The integrated intensity of the Bragg peaks is proportional to the quantity of bound protein in ordered clusters with $n=0$ and $n=1$ showing the largest amount, $n=3$ a smaller amount, and with no ordered clusters observed for $n=7$ linkers. To calculate the relative fractions of protein in ordered clusters, integrated intensities of the protein Bragg peaks were normalized by the protein layer coverage. Resulting values, scaled to 1 for $n=0$, show that increasing linker length decreases the fraction of clustered STxB that contributes to the GIXD signal (main text Figure 2, red squares). This is consistent with measurements of $\mathrm{STxB}$ in contact with or at small separations from the membrane reducing the spectrum of membrane fluctuations between neighboring proteins thereby driving them into contact ${ }^{2}$. However, in this work the monolayer was confined to a planar geometry at the air-water interface which likely severely restricts its fluctuation spectrum. This suggests that another mechanism dependent on relatively weak protein-protein interactions may contribute to the in-plane clustering of STxB, particularly under the high surface coverage experimental conditions used here.

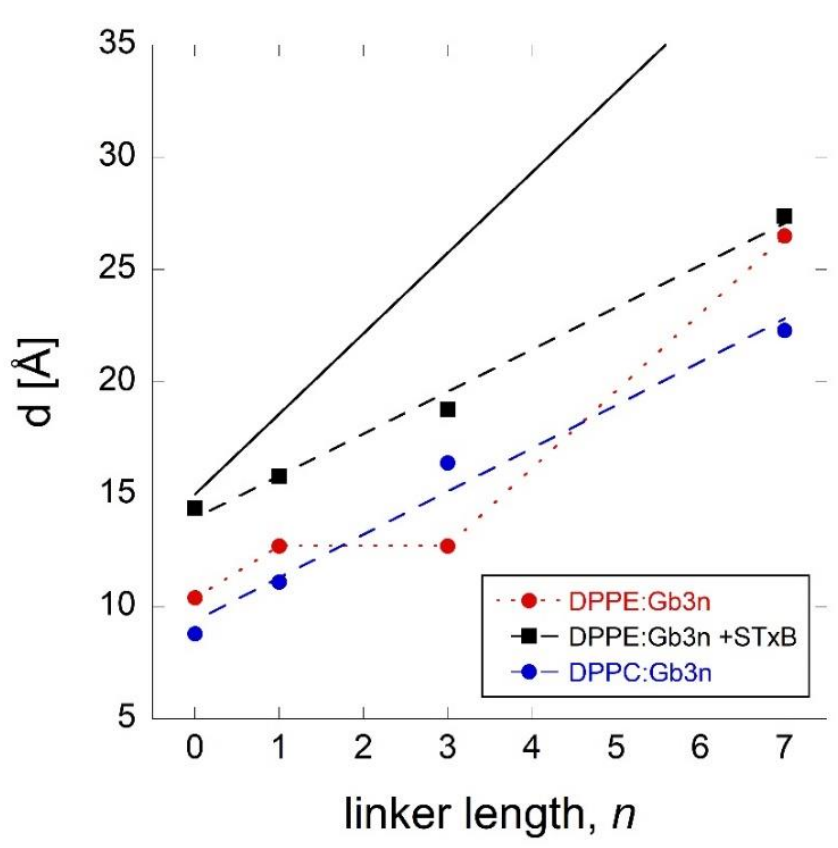

Figure S-4. Comparison of Gb3 extension from the membrane plane in DPPE:Gb3 ${ }_{n}$ (red circles) and DPPC:Gb3 ${ }_{n}$ (blue circles) membranes with the location of bound STxB relative to the membrane (black squares). The solid black line corresponds to the maximum possible extension of the Gb3 carbohydrate. The non-linear dependence of the carbohydrate extension in DPPE:GB3 ${ }_{n}$ membranes indicates an influence of PEG linkers on Gb3 carbohydrates. This influence is not apparent following STxB binding or in DPPC membranes. Dashed black and blue lines are linear fits to the data points and the red dotted line is a guide for the eye.

Influence of PEG linkers on Gb3 carbohydrate conformation

Quantitative information about the influence of PEG linker length on Gb3 carbohydrate conformation was obtained using XR. In general, while the PEG linkers can influence the average Gb3 carbohydrate conformation these changes do not significantly impact STxB binding. We distinguish between two distinct effects of the PEG linkers on Gb3 conformation: (1) how the Gb3 carbohydrate orients with respect to the lipids within the membrane, and (2) how the PEG linker influences carbohydrate presentation and availability for STxB binding.

XR measurements of the lipid:Gb3 monolayers were used to determine the average extension of the carbohydrate away from the membrane plane. Results of this analysis are shown in Figure S-4 where $d$ is the distance between the lipid head groups and the most extended region of the Gb3 carbohydrate. The black line corresponds to the 
maximum possible extension of the carbohydrate. This was determined by summing the length of an extended carbohydrate conformation obtained from a Universal Force Field energy minimization (15 $\AA$ ) and the length of a fully extended PEG chain with monomer length of $3.58 \AA$. The red points correspond to experimentally measured Gb3 carbohydrate extension in DPPE:Gb3 ${ }_{n}$ monolayers in the absence of $\mathrm{STxB}$. In general, the results show a fairly extended conformation of the carbohydrate. Interestingly, there is a significant deviation from a linear dependence of carbohydrate extension on PEG linker length at $n=3$. This suggests a conformational change of the carbohydrate that may depend on the PEG linker. This may be analogous to previously observed conformational changes in GSL carbohydrates in the presence of cholesterol ${ }^{10}$. As in the case of cholesterol, DPPE has negative spontaneous curvature which, in a flat monolayer, may result in unfavorable exposure of the hydrophobic tails to water and thereby promote carbohydrate bending. We hypothesize that the conformational change observed for $n=3$ linkers involves bending of the Gb3 to shield DPPE tails from solvation. For $n=0$ and $n=1$, there was not sufficient flexibility to enable bending, and for $n=7$ an extended conformation was more entropically favorable. In contrast, the blue points, obtained from analysis of $\mathrm{XR}$ data on DPPC:Gb3 ${ }_{n}$, shows a linear dependence of $d$ on linker length. Since DPPC does not have negative spontaneous curvature, there is likely no driving force to induce carbohydrate bending in order to shield the lipid tails from water. Our analysis of the DPPE:Gb3 ${ }_{n} \mathrm{XR}$ results shows that for the $n=3$ linkers, the Gb3 carbohydrate has a preferred orientation, when averaged over the measurement time, that shields the DPPE tails. However, we believe that the PEG linker still enables the carbohydrate to sample a wider configurational space including a set of conformations that allow STxB binding.

Despite the influence of PEG linkers on the Gb3 carbohydrate conformation, similar quantities of protein binding were observed for all linker lengths. In fact, for the $n=3$ linker, which exhibits a less extended carbohydrate conformation on average, there was slightly more protein bound than for the other linkers. This demonstrates that any effect of the PEG linker on Gb3's carbohydrate conformation does not significantly impact STxB binding. We hypothesize that this is due to the fact that, even if there is a preferred carbohydrate conformation that makes the receptor less accessible to binding, the PEG linker still enables the carbohydrate to explore other conformations that are more favorable to protein binding. This is demonstrated by the linear dependence of the protein spacing on linker length plotted the distance from the lipid head groups to the center of mass of the protein as a function of linker length (Figure $S$ 4 , black squares) which shows that after binding specific preferential conformations of the carbohydrate relative to the membrane plane are eliminated. Overall, these results suggest that increasing the linker length increases the possible orientations that the sugars are presented. In addition, PEG linkers increase separation between the carbohydrate and the phospholipid head groups which should alleviate steric hindrances on the Gb3 conformation.

Estimation lateral distance of long-range lipid reorganization propagation
Despite partial protein coverage, after STxB binding all lipids in the monolayer exhibited a condensed packing. This indicates that lipid reorganization propagates laterally across protein-free membrane domains. While these experiments are unable to directly obtain the characteristic size of protein-free domains, estimates and some bounds on their dimensions can be extracted from the average protein coverage obtained from XR and the characteristic size of the protein clusters obtained from GIXD. One approach to estimate a minimum distance for lipid order propagation is to assume that the lipid APM reduction requires interaction with protein clusters (consistent with the $n=7$ results) and that the clusters are circular and arranged on a hexagonal lattice (with a minimum spacing $a_{c}$ between clusters). For the $\mathrm{n}=0$ case, the $76 \mathrm{~nm}$ coherence length obtained from GIXD (Table S-2) corresponds to circular domains with an approximate cluster area of $4,500 \mathrm{~nm}^{2}$. Assuming that all bound proteins are in clusters, these clusters would take up $25 \%$ of the surface and the area of a hexagonal unit containing one cluster is $18,000 \mathrm{~nm}^{2}$. The lattice parameter, $a_{c}$, for this unit cell is the distance between clusters and can be calculated using simple geometry:

$$
a_{c}=\sqrt{\frac{18000}{0.866}}=144 \mathrm{~nm}
$$

Subtracting the $76 \mathrm{~nm}$ cluster diameter yields a distance of $68 \mathrm{~nm}$ between the outer edges of two clusters. Considering that only a fraction of the total quantity of bound proteins is in clusters, the remaining isolated proteins would occupy the space between clusters. Therefore, the size of the unit cell and the distance between clusters will increase with the fraction of isolated protein. These estimations indicate a minimum propagation distance of 10 s of nanometers for the lipid reorganization. Since it is also possible that larger length scale regions (i.e. microns) of enhanced/depleted protein concentration exist, this estimate represents a lower bound for the propagation distance.

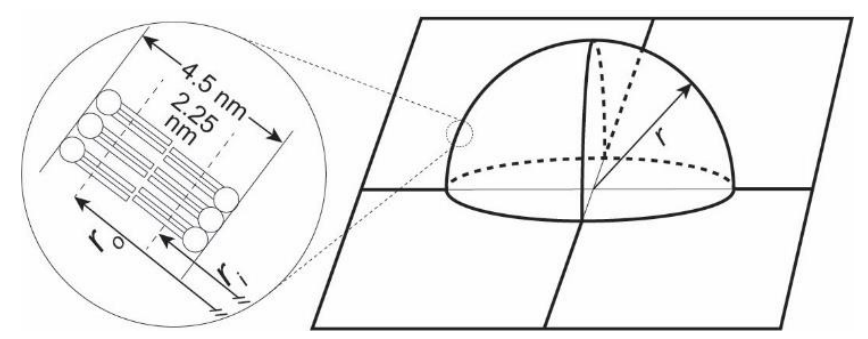

Figure S-5. Schematic of a hemispherical deformation in a planar membrane. The radius of curvature of the invagination is determined by the magnitude of lipid APM condensation in the inner leaflet.

Calculation of the radius of curvature resulting from asymmetric leaflet compression

Changes in the area per lipid of one leaflet of a bilayer relative to the other can result in bending of the membrane such that the surface 
areas of each leaflet compensate for the asymmetric change in areas. Here, we present a simplified continuum level description of how asymmetric leaflet compression can generate a hemispherical deformation in a planar membrane and relate the radius of curvature to the ratio of leaflet areas. Such a hemispherical deformation can be considered as an initial precursor for the subsequent formation of a tubular invagination. First, we take the total thickness of a lipid bilayer, measured from the outer faces of each leaflet's head groups, to be approximately $4.5 \mathrm{~nm}$. This makes the spacing between the midplanes of each leaflet approximately $2.25 \mathrm{~nm}$ and therefore, for a curved membrane, the radius of curvature of the inner $\left(r_{i}\right)$ and outer $\left(r_{o}\right)$ leaflets differs by $2.25 \mathrm{~nm}$. For a hemi-spherical membrane deformation, the integrated surface area of each leaflet is $A=2 \pi r^{2}$ where $r$ is $r_{i}$ and $r_{0}$ for the inner and outer leaflets respectively (Figure S-5). The minimum radius of curvature of such a deformation corresponds to a hemisphere where the ratio between the leaflet areas matches the ratio of the lipid APMs before and after STxB binding: $\mathrm{APM}_{\mathrm{o}} / \mathrm{APM}_{\mathrm{i}}=2 \pi r_{o}^{2} / 2 \pi r_{i}^{2}$. For $r_{o}=r_{i}+2.25 n m$, we obtain $r_{i}=$ $\frac{2.25}{\sqrt{\frac{A P M_{O}}{A P M_{i}}}-1} n m$. Using this approach, the 0.3-0.8\% APM condensations observed have the potential to form $\sim 500-1500 \mathrm{~nm}$ radius of curvature membrane invaginations which could serve to initiate the formation of membrane tubules. See discussion in the main manuscript on corresponding radii in fluid membranes.

\section{REFERENCES}

1. Johannes, L. Toxins 2017, 9, (11).

2. Pezeshkian, W.; Gao, H. F.; Arumugam, S.; Becken, U.; Bassereau, P.; Florent, J. C.; Ipsen, J. H.; Johannes, L.; Shillcock, J. C. Acs Nano 2017, 11, (1), 314-324.

3. Pezeshkian, W.; Hansen, A. G.; Johannes, L.; Khandelia, H.; Shillcock, J. C.; Kumar, P. B. S.; Ipsen, J. H. Soft Matter 2016, 12, (23), 5164-5171.

4. Roemer, W.; Berland, L.; Chambon, V.; Gaus, K.; Windschiegl, B.; Tenza, D.; Aly, M. R. E.; Fraisier, V.; Florent, J.-C.; Perrais, D.; Lamaze, C.; Raposo, G.; Steinem, C.; Sens, P.; Bassereau, P.; Johannes, L. Nature 2007, 450, (7170), 670-U3.

5. Abeles, F. J Phys-Paris 1950, 11, (7), 307-309.

6. Parratt, L. G. Physical Review 1954, 95, (2), 617-617.

7. Perkins, S. J. European Journal of Biochemistry 1986, 157, (1), 169-180.

8. Watkins, E. B.; Gao, H.; Dennison, A.J. C.; Chopin, N.; Struth, B.; Arnold, T.; Florent, J.-C.; Johannes, L. Biophysical Journal 2014, 107, (5), 11461155.

9. Patterson, A. L. Physical Review 1939, 56, (10), 978-982.

10. Lingwood, D.; Binnington, B.; Rog, T.; Vattulainen, I.; Grzybek, M.; Coskun, U.; Lingwood, C. A.; Simons, K. Nat Chem Biol 2011, 7, (5), 260262. 